\title{
Mündliche familiale Interaktionsmuster und schriftliche argumentative Textproduktion im Verlauf der Sekundarstufe I
}

\begin{abstract}
Dieser Beitrag untersucht das Zusammenspiel mündlicher familialer Interaktionsmuster und schriftlicher argumentativer Textproduktion im Verlauf der Sekundarstufe I. Als Datengrundlage dient das einzigartige längsschnittliche Korpus mündlicher und schriftlicher Familien- und Schülerdaten sowie standardisierter Hintergrundinformationen der sogenannten Intensivstichprobe des Projekts FUnDuS. Über die Kombination qualitativer und quantitativer methodischer Zugänge sollen $\mathrm{u}$. a. folgende Forschungsfragen bearbeitet bzw. beantwortet werden: Wie entwickelt sich die schriftliche (persuasive und explorative) argumentative Textproduktion im Verlauf der Sekundarstufe I 1) je nach familialem Interaktionsmuster zu Beginn der Sekundarstufe I und 2) bei Kontrolle familialer bzw. individueller Ausgangsbedingungen (sozioökonomischer Status bzw. Intelligenz)?
\end{abstract}

Keywords: Mündliche Interaktionsmuster, schriftliches Argumentieren, Sekundarstufe I, sozioökonomischer Status, kognitive Vorläuferfähigkeiten

\section{Einleitung}

Argumentative Fähigkeiten zählen nicht ohne Grund zu den Schlüsselkompetenzen für gesellschaftliche Teilhabe: Als zentrale Diskursfunktion (Feilke 2013; Vollmer 2011) prägen sie sowohl private und öffentliche Gespräche und werden in den Curricula als wesentliche Dimension sprachlicher und nicht-sprachlicher Fachleistungen genannt (KMK 2004a; KMK 2004b). Gerade im Verlauf der Sekundarstufe wird diese bildungssprachlich einschlägige Praktik (Morek \& Heller 2012) zunehmend medial schriftlich realisiert.

Die Ausbildung argumentativer Kompetenzen im Kindes- und Jugendalter scheint - wie andere schulisch relevante Fähigkeiten auch - von verschiedenen familiären und individuellen Hintergrundbedingungen abzuhängen. Verschiedene

Madeleine Domenech, Universität Paderborn, Warburger Str. 100, 33098 Paderborn, madeleine.domenech@uni-paderborn.de

Antje Krah, Technische Universität Dortmund, Fakultät Kulturwissenschaften, antje.krah@tu-dortmund.de

○ Open Access. (C) 2021 Madeleine Domenech, et al., publiziert von De Gruyter. (c))BY-NC-ND Dieses Werk ist lizensiert unter einer Creative Commons Namensnennung - Nicht-kommerziell - Keine Bearbeitung 4.0 International Lizenz. 
Studien belegen einerseits die Relevanz der ,üblichen Verdächtigen“, wie den soziokulturellen Hintergrund oder den (solche Faktoren kumulierenden) Bildungsgang der Schülerinnen und Schüler (Krelle \& Willenberg 2008; Neumann \& Lehmann 2008; Quasthoff et al. 2015). Andererseits verweisen einige Befunde auch auf das Potenzial kommunikativer Ressourcen im Elternhaus für die Entwicklung von Argumentationskompetenz in der Sekundarstufe I - gerade auch neben bzw. unabhängig von anderen Ressourcen, wie der sozialen Herkunft oder relevanten Vorläuferfähigkeiten (Krah et al. 2013; Quasthoff et al. 2015; Quasthoff et al. 2016). In diesem Zusammenhang scheinen insbesondere bestimmte Mechanismen und Muster der Eltern-Kind-Interaktionen eine besonders erwerbsunterstützende Rolle zu spielen (Hausendorf \& Quasthoff 1996, 2005; Quasthoff \& Kern 2007).

Genau an dieser Schnittstelle setzt der vorliegende Beitrag an, indem er die Bedeutung kommunikativer Ressourcen im Elternhaus am Beispiel familialer Interaktionsmuster für die Entwicklung argumentativer Fähigkeiten (hier: schriftliches Argumentieren) im Verlauf der Sekundarstufe I untersucht.

Abschnitt 2 fasst in der gebotenen Kürze Forschungen zur Rolle familialer Interaktionsmuster bei der Ausbildung von Argumentationskompetenz im Jugendalter zusammen. Der Abschnitt 3 stellt die Anlage der Untersuchung vor; Abschnitt 4 widmet sich anschließend der Darstellung bzw. Interpretation der Ergebnisse. Diese zeigen eine variierende Erwerbssupportivität verschiedener Muster von Eltern-Kind-Interaktionen in Bezug auf die Qualität schriftlicher Argumentationen in den Klassen 6, 7 und 9. Schließlich fasst Abschnitt 5 die Befunde rückblickend zusammen bzw. perspektiviert sie für zukünftige Untersuchungen.

\section{Bestandsaufnahme: Die Rolle familialer Interaktionsmuster bei der Ausbildung von Argumentationskompetenz}

Um Interaktionsmuster rekonstruieren zu können, wurde das Instrument GLOBE (Hausendorf \& Quasthoff 2005) auf Grundlage von Erzählungen entwickelt, das es ermöglicht, verschiedene Interaktionen miteinander vergleichbar $\mathrm{zu}$ machen (vgl. zu diesem und anderen grundlegenden Konzepten das Glossar in diesem Band). Es dient bei der Analyse als Tertium Comparationis und wurde bereits auch für andere Gattungen adaptiert (für Erklärungen: Morek 2012 und Quasthoff \& Kern 2007, für Argumentationen: Heller 2012). Mit Hilfe dieses Beschreibungsinstrumentes können sprachliche und nonverbale Formen beschrieben werden, 
mithilfe derer pragmatische Mittel realisiert werden, die zur Erledigung eines kommunikativen Jobs von den Interagierenden genrespezifisch genutzt werden. So kann im Falle einer Argumentation (siehe auch Domenech, Heller \& Petersen 2018) z. B. weil (sprachliche Form) genutzt werden, um eine Begründung (Mittel) einzuleiten, um eine These zur Disposition zu stellen (Job). Je nachdem wie stark die Kinder an den jeweiligen Jobs partizipieren und wie die Eltern dies interaktiv unterstützen und steuern können, werden dann in einem nächsten Schritt ähnliche Praktiken zu Mustern zusammengefasst.

Der Einfluss familialer Kommunikationsmuster in Bezug auf Argumentationskompetenz wurde bisher insb. im Kontext des Forschungsprojekts Die Rolle familialer Unterstützung beim Erwerb von Diskurs- und Schreibfähigkeiten in der Sekundarstufe I (FUnDuS I und II) untersucht, welches sich in seiner Laufzeit von 2009-2016 der Identifikation relevanter familialer Faktoren zur Erklärung argumentativer Fähigkeiten gewidmet hat. Hierfür wurden Schülerinnen und Schüler sowie Eltern von der fünften bis zur neunten Klasse im Rahmen eines komplexen triangulativen longitudinalen Designs begleitet (siehe z. B. Krah et al. 2013; Wild et al. 2012; Quasthoff, Wild et al. in diesem Band für eine detaillierte Beschreibung). Dieses vereint sowohl unterschiedliche fachliche (Psychologie und Linguistik) und methodische (quantitative und qualitative) Zugriffe als auch verschiedene Datentypen (Fragebogenangaben, schriftliche Schülertexte, mündliche ElternKind-Interaktionen).

\subsection{Zentrale familiale, mündliche Interaktionsmuster}

Im Rahmen der qualitativen Analysen mündlicher Eltern-Kind-Interaktionen von FUnDuS konnten drei zentrale Interaktionsmuster gesprächsanalytisch rekonstruiert werden: Fordern und Unterstützen, Raumlassen und Akzeptieren, Übergehen und Selberlösen. Diese Muster fokussieren einerseits Steuerungsmechanismen der Eltern und andererseits die kindlichen Anteile an der jeweiligen Interaktion und werden zur besseren Nachvollziehbarkeit im Folgenden an exemplarischen Transkriptausschnitten aus Erhebungszeitpunkt (EZP) 1 der Intensivstichprobe kurz illustriert (siehe auch Heller 2012; Quasthoff \& Krah 2015). In diesem Fall werden die Muster jedoch nicht auf Grundlage der mündlichen Argumentationsaufgabe (s. Quasthoff \& Kluger in diesem Band) rekonstruiert, sondern auf Basis der Eltern-Kind-Interaktionen zu den von den Kindern verfassten Texten. Das Szenario der Texte basiert dabei auf einer Detektivgeschichte, in der das Kind ein Kommissar ist und an einem ,Tatort' (das Handy 
von Herrn Huber ist verschwunden) feststellt (vgl. auch Abb. 2, Abschnitt 3.1.1: Aufgabe zu EZP 2):

1) der Hund verbuddelt eine Fernbedienung im Garten,

2) der Nachbarsjunge Tim war gestern ganz traurig, weil er sein Handy verloren hat und heute ist er wieder fröhlich und

3) die Nichte war gestern zu Besuch und auf dem Sofa im Wohnzimmer liegt ein Handy, das nicht Herrn Huber gehört.

Das Muster Fordern und Unterstützen zeichnet sich u. a. dadurch aus, dass das Kind als primärer Aufgabenlöser etabliert wird (SO = Sohn, MU = Mutter):

Bsp. 1: Fall 1113 UE $1^{1}$

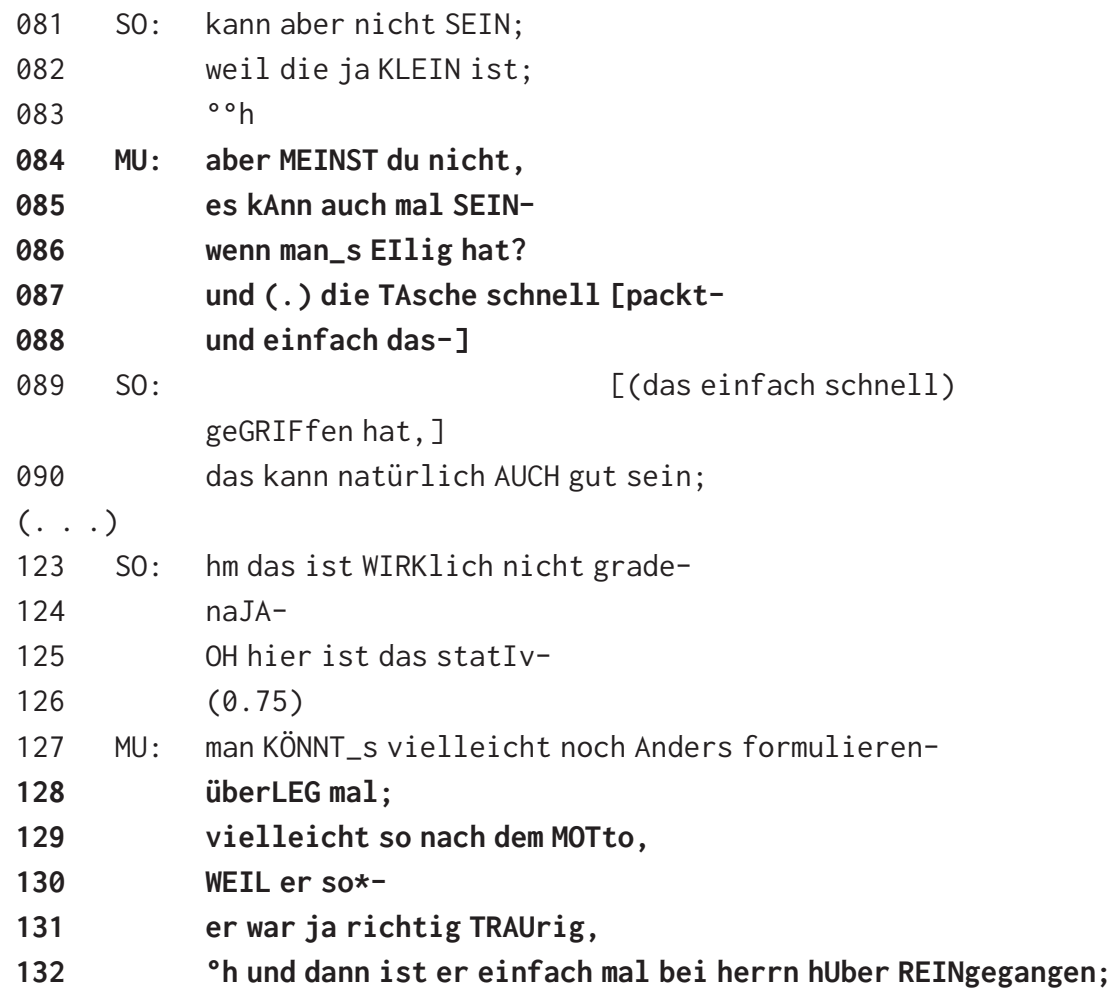

1 Bei den Beispielen handelt es sich um die Revision der geschriebenen Texte der Kinder. Elternteil und Kind diskutieren über den Fall und darüber, wie der Bericht an den Chef „noch besser gemacht“ werden kann. Die Transkripte folgen der Notation von GAT 2 (s. Anhang zu diesem Band). Herauszuhebende Passagen erscheinen in Fettdruck. 
133

134

135

136

137 meinst_e er ist GLEICH (. ) reingegangen, [weil er (.) das HANdy klauen wollte?]

SO: [ey genAU-

du hast mich jetzt-

ja das ist GUT; ]

Die Eltern fordern ihr Kind, indem sie z. B. Einwände formulieren, die beim Kind die Berücksichtigung weiterer Tathergänge innerhalb des Detektivszenarios auslösen. Das Kind ( $\mathrm{SO}=\mathrm{Sohn}$ ) bekommt hier die Gelegenheit, weitere Perspektiven zu berücksichtigen und Gegenargumente zu entwickeln. In diesem Beispiel schafft die Mutter dies, indem sie das Szenario des Sohnes in einem kleinen Punkt in Frage stellt: dass man das falsche Handy u. U. doch einsteckt, wenn man es eilig hat (Z. 084-088). Zudem eröffnet der Elternteil dem Kind auf diese Weise Gesprächsraum, den das Kind zur weiteren Lösung der Aufgabe nutzen kann. Zusätzlich unterstützt die Mutter ihren Sohn an einer Stelle, an der er selbst nicht mehr weiterkommt und gibt ihm eine Idee und eine Frage mit (Z. 128-134). Diese werden direkt vom Sohn als produktiv und weiterführend bewertet (Z. 135-137) und er kann im Folgenden selbst weiter formulieren.

Im Gegensatz dazu fordern die Eltern, die das Muster Raumlassen und Akzeptieren verwenden, ihre Kinder nicht, sondern pflichten ihnen in ihren Überlegungen bei, ohne die Argumente in Frage zu stellen (TO = Tochter, MU = Mutter):

\section{Bsp. 2: Fall 244 UE 1}

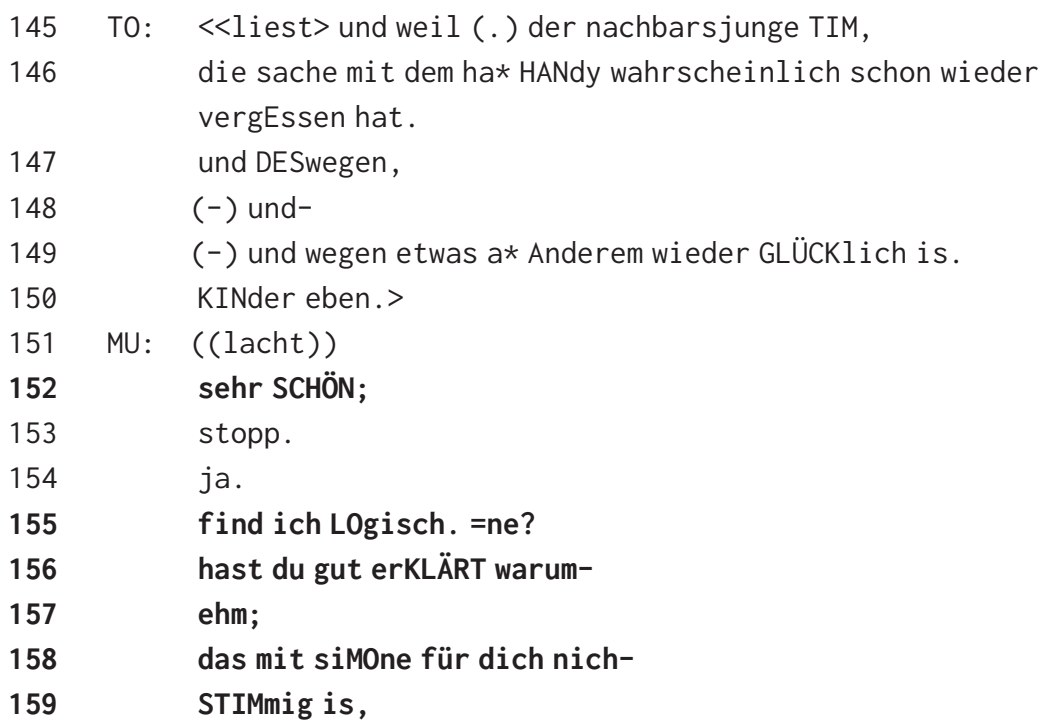


Hier wird dem Kind Gesprächsraum zugestanden, der vergleichsweise frei gefüllt werden kann. Der Elternteil stellt keinen Dissens her oder löst ihn, wie in anderen Gesprächen zu beobachten, selbst auf. Somit werden dem Kind keine neuen kommunikativen Aufgaben gestellt, in denen es Gegenargumente oder Perspektiven entwickeln müsste. In Gesprächen dieses Typs entsteht i.d.R. eher eine Sammlung von Gründen als ein globaler Argumentationszusammenhang.

Das Muster Übergehen und Selberlösen zeichnet sich dadurch aus, dass ein Elternteil die Rolle des Aufgabenlösers übernimmt (Z. 047-049) und sich selbst als Kundiger/Wissender etabliert (VA = Vater, $\mathrm{SO}=\mathrm{Sohn})$ :

\section{Bsp. 3: Fall 789 UE 1}

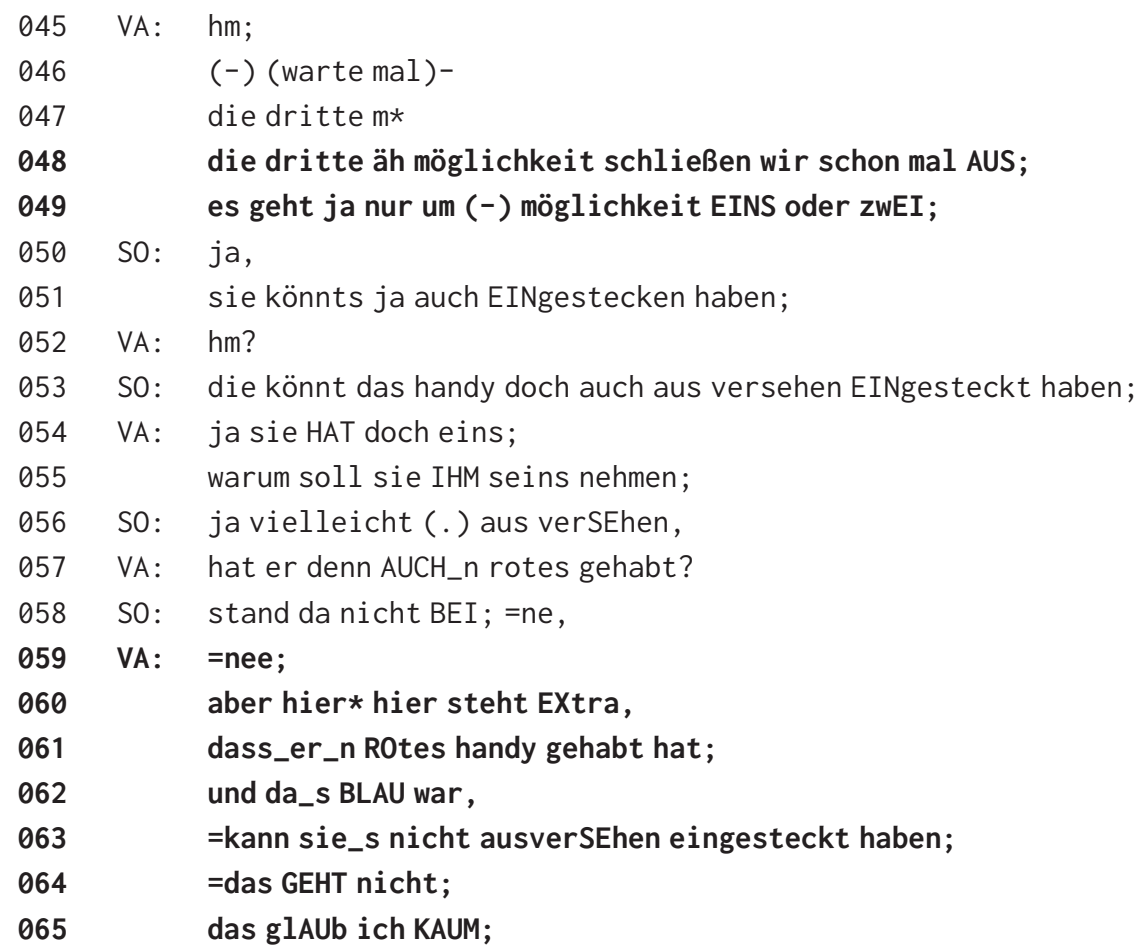

In Beispiel 3 ,löscht‘ der Vater sogar die Begründungen des Sohnes, indem er die Argumentation als inkorrekt darstellt (Z. 059). Dem Kind wird somit die Kompetenz zur Bekundung und Begründung einer eigenen Position abgesprochen. 
Anstelle von Begründungen realisiert der Elternteil hier eine Belehrung (Z. 060065), indem er einerseits auf die Aufgabe referiert (Z. 060) und damit seinem Sohn abspricht, die Aufgabe richtig gelesen und verstanden zu haben, und andererseits die Möglichkeit der Verwechslung aufgrund unterschiedlicher Farben der Handys ausschließt (Z. 061-063). In anderen Fällen wird das Kind als Stichwortgeber etabliert oder ihm wird der Gesprächsraum gar verweigert.

Die beschriebenen Interaktionsmuster traten in den Familien unabhängig von ihrem sozioökonomischen Status auf und waren über die drei EZPe konstant. Lediglich das Muster Übergehen und Selberlösen wurde in sozial privilegierten Familien bei hoher Argumentationskompetenz des Kindes nicht verwendet (Heller \& Krah 2015; Quasthoff et al. 2015; Quasthoff et al. 2016).

\subsection{Befunde zur Erwerbssupportivität von Interaktionsmustern auf die Ausbildung argumentativer Fähigkeiten}

Den Einfluss dieser Interaktionsmuster auf die Ausbildung übergreifender argumentativer Fähigkeiten untersuchen Domenech, Krah \& Hollmann (2017). Hierfür kontrastieren sie mit Hilfe latenter Wachstumskurvenanalysen die Entwicklung der Argumentationskompetenz von Schülerinnen und Schüler aus Familien, die das Muster Fordern und Unterstützen praktizieren, mit solchen, deren Eltern Übergehen und Selberlösen anwenden. Dabei zeigt sich erwartungskonform für die Gruppe Fordern und Unterstützen ein höheres Ausgangsniveau sowie ein bedeutsamer Kompetenzzuwachs von Klasse 6 zu Klasse 9 gegenüber der niedrig ,startenden' und sich kaum entwickelnden Gruppe Übergehen und Selberlösen.

Erste Thesen zur Wirksamkeit der Interaktionsmuster in Bezug auf die jeweiligen Ausprägungen der Dimensionen von mündlicher Argumentationskompetenz arbeiten Heller \& Krah (2015) heraus und stellen den Zusammenhang her, dass das Muster Fordern und Unterstützen global gesehen die meisten Chancen für die Ausbildung mündlicher argumentativer Fähigkeiten bietet. Hingegen scheinen Kinder beim Muster Raumlassen und Akzeptieren vor allem Vertextungsverfahren (s. Heller in diesem Band) ausbauen zu können. Dadurch, dass das Kind beim Muster Übergehen und Selberlösen nur unwesentlich in die Interaktion mit einbezogen wird, kann es allenfalls seine Kontextualisierungskompetenz (Quasthoff 2009; Quasthoff 2011) ausbauen, indem es eigeninitiativ in die Interaktion einsteigt. Auch Quasthoff \& Kluger (in diesem Band) bestätigen, dass die Kinder im Muster Fordern und Unterstützen über den Längsschnitt eine Verbesserung des eigenständigen Argumentierens zeigen. Beim Muster Raumlassen und Akzeptieren scheint jedoch eher die Kompetenz des Kindes bedeutend zu sein, inwieweit es in der Lage ist, Räume, die ihm gegeben werden, 
eigenständig zu nutzen. Für Kinder im Muster Übergehen und Selberlösen zeichnet sich kein Aufwärtstrend in Bezug auf eigenständigeres Argumentieren ab.

Zum Zusammenhang von mündlichen Interaktionsmustern und argumentativer Textproduktion(en) im Schriftlichen liegt bisher nur die Untersuchung von Domenech \& Krah (2014) vor. Am Beispiel zweier systematisch ausgewählter Einzelfälle der FUnDuS-Stichprobe aus Klasse 5 wird hier zum einen erneut die eingeschränkte Erklärungskraft bzw. Varianzaufklärung des sozioökonomischen Status (im Folgenden: SES) für übergreifende und schriftliche argumentative Fähigkeiten illustriert. Zum anderen verweisen die Analysen auch für das Schriftliche auf die Erwerbssupportivität des Musters Fordern und Unterstützen im Vergleich zu Übergehen und Selberlösen.

Wie diese kurze Zusammenschau verdeutlicht, ist die Rolle familialer Interaktionsmuster insb. für das schriftliche Argumentieren bisher also vergleichsweise wenig erforscht. ${ }^{2}$ So liegen weder Untersuchungen vor,

- die sich auf größere Stichproben beziehen,

- die den Entwicklungsverlauf in den Blick nehmen,

- die alle drei Interaktionsmuster (s. o.) einbeziehen oder

- die die ,üblichen verdächtigen“ familialen bzw. individuellen Ressourcen wie SES oder kognitive Vorläuferfähigkeiten systematisch berücksichtigen.

Genau diese Desiderate greifen die empirischen Analysen des vorliegenden Beitrags auf, wie nachfolgend erläutert.

\section{Anlage der Untersuchung}

Auf die oben formulierten Desiderate bezugnehmend untersucht der vorliegende Beitrag folgende empirische Fragestellung:

- Wie entwickelt sich die schriftliche argumentative Textproduktion im Verlauf der Sekundarstufe I in Abhängigkeit familialer Interaktionsmuster und bei Kontrolle familialer bzw. individueller einschlägiger Ausgangsbedingungen?

Auch wenn die Befundlage zum schriftlichen Argumentieren wie oben (2.2) dargestellt noch sehr lückenhaft ist, wäre vor dem Hintergrund der bisher vorliegenden

2 Vergleichsweise bezieht sich in diesem Fall sowohl auf die tendenziell häufigere Bearbeitung des Zusammenhangs familialer Interaktionsmuster mit mündlichen Fähigkeiten als abhängiger Variable als auch auf die überraschende Nicht-Berücksichtigung dieser unabhängigen Variable für die ansonsten breit erforschte(n) Herausbildung(sbedingungen) argumentativer Fähigkeiten im Schriftlichen. 
Ergebnisse zum Zusammenhang familialer Interaktionsmuster mit argumentativen Fähigkeiten zu erwarten, dass sich die Texte von Schülerinnen und Schülern aus Familien, welche fordern und unterstützen, durch eine insgesamt höhere Textqualität auszeichnen gegenüber denjenigen, deren Eltern übergehen und selberlösen. Auf Grund der dargestellten Untersuchungsergebnisse und -thesen zum Erwerb mündlicher Argumentationskompetenz im Muster Raumlassen und Akzeptieren könnten Kinder profitieren, die ein ohnehin besseres Startniveau haben.

\subsection{Datengrundlage und Operationalisierung}

Die zur Beantwortung der Fragestellung (s. o.) durchzuführenden empirischen Analysen stützen sich auf folgende Daten und Operationalisierungen des Forschungsprojekts FUnDuS (siehe Krah et al. 2013; Quasthoff et al. 2015 für detailliertere Beschreibungen des Designs von FUnDuS).

\subsubsection{Schriftliche Argumentation}

Die Maße für die schriftliche argumentative Textproduktion stützen sich auf etablierte Aufgabentypen der Schreibforschung, nämlich eine persuasive und eine explorative argumentative Schreibaufgabe (z. B. Augst \& Faigel 1986; Augst et al. 2007; DESI; Rezat 2011). Während es bei der Persuasion darauf ankommt, sein Gegenüber von der eigenen Sichtweise zu überzeugen, müssen bei der Exploration verschiedene Positionen begründend gegeneinander abgewogen werden (Ehlich et al. 2012). ${ }^{3}$ Die persuasive Aufgabe ist in ein Szenario eingebettet, in dem es um ein Schulfest geht. Die Schülerinnen und Schüler sollen ihren zurzeit kranken Schulkameraden Christian in einem Brief davon überzeugen, dass die ganze Klasse - ihn eingeschlossen - mitmachen soll. Christian will am Schulfest nämlich nicht teilnehmen (Brief an Christian). ${ }^{4}$

3 Für weitere Unterscheidungen des Argumentierens siehe auch Becker-Mrotzek \& Böttcher 2012; Winkler 2003.

4 Diese Aufgabe blieb über den gesamten Erhebungszeitraum unverändert. 


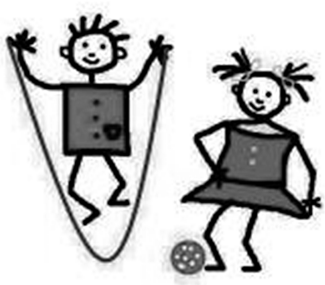

Nun stellt euch folgende Situation vor: Endlich wird es Frühling und eure Schule plant für einen Nachmittag ein großes Schulfest, worauf ihr euch alle sehr freut. Jede Klasse, die teilnimmt, muss sich dafür etwas Besonderes ausdenken. Sie kann z.B. Kuchen verkaufen, aus dem Klassenzimmer eine Geisterbahn machen, eine KaraokeShow für die Lehrer veranstalten, usw.. Die Teilnahme ist freiwillig. Eure Klasse kann mitmachen, muss aber nicht.

Warum sollte eure Klasse beim Schulfest mitmachen? Wieso solltet ihr etwas anbieten?

Schreibt in einem Satz einen möglichst guten Grund auf!

$\rightarrow$

Christian, ein Mitschüler von euch, hat keine Lust am Schulfest teilzunehmen. Ihr wollt ihn am nächsten Tag davon überzeugen, dass eure Klasse unbedingt mitmachen sollte. Christian ist aber leider krank. Ihr schreibt ihm deshalb einen Brief. Euer Lehrer soll den Brief hinterher unterschreiben und an Christian schicken. Ihr wollt Christian überzeugen, indem ihr möglichst gute Gründe nennt.

Was würdet ihr in dem Brief schreiben? Ihr dürft hier auch den Grund benutzen, den ihr euch eben überlegt habt. Denkt daran: Es geht darum, Christian mit möglichst guten Gründen zu überzeugen!

Abb. 1: Schreibaufgabe Brief an Christian. 


\section{Aufgabe}

Vor einem Jahr hast Du den Fall von Herrn Hubers verschwundenem Handy gelöst. Dein Chef, Kommissar Klöbner, hat nun einen neuen Fall für Dich:

Frau Schuster hat ihre Katze vermisst gemeldet. Sie hat sie seit drei Tagen nicht mehr gesehen.

Du stellst Nachforschungen vor Ort an und erfährst Folgendes:

1. Die Katze von Frau Schusters Nachbarn gegenüber, Familie Meier, ist vor ein paar Wochen gestorben. In deren Garten steht jedoch ein Schälchen mit Katzenfutter.

2. Der Keller von Frau Schusters Haus ist verschlossen. Frau Schuster erklärt, dass sie den Schlüssel vor ein paar Tagen verlegt hat.

3. Frau Schuster sagt, dass ihre Katze gern in der Garage spielt. Die ist allerdings leer, weil ihr Neffe sich vor drei Tagen ihr Auto geliehen hat, um damit in den Urlaub zu fahren.

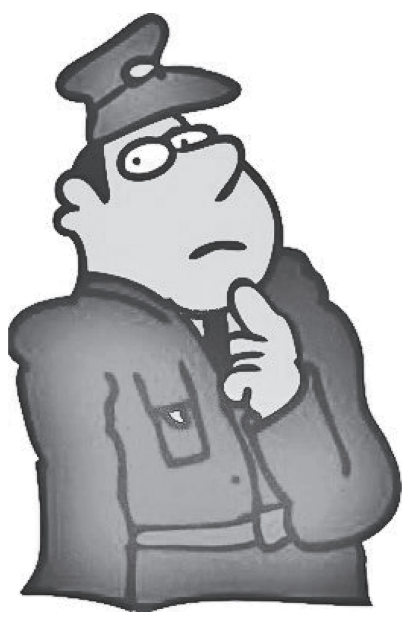

Du denkst über diese Hinweise nach und hast mehrere Ideen, was passiert sein könnte. Schließlich glaubst Du den Fall aufgeklärt zu haben, weil Du weißt, wie die Katze verschwunden ist.

Schreib einen Bericht an Deinen Chef, in dem $\mathrm{Du}$ Deine Meinung zu dem Fall genau begründest. Gib dabei auch an, warum Du andere Möglichkeiten für weniger wahrscheinlich hältst.

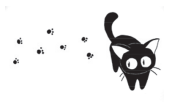

Abb. 2: Schreibaufgabe Brief an den Kommissar zu EZP 2. 
In der explorativen Aufgabe sollen die Kinder - wie erwähnt - in einem fiktiven Szenario an einem ,Tatort' als Detektiv ermitteln. Dabei werden jeweils drei Indizien vorgegeben, aus denen sie den für sie selbst wahrscheinlichsten Tathergang rekonstruieren sollen. Dieser soll in einem Bericht an den Chef, Kommissar Klöbner, unter Ausschluss der anderen Möglichkeiten begründend dargestellt werden (Bericht an den Kommissar).

Die Auswertung der zwei argumentativen Schreibaufgaben erfolgte für die Klassen 6, 7 und 9 auf Basis eines Verfahrens zur Erfassung von Textqualität (TexQu), welches im Kontext von FUnDuS theoriebasiert entwickelt und empirisch erprobt wurde (Quasthoff \& Domenech 2016). ${ }^{5}$ TexQu fußt auf einem medialitätsund gattungsübergreifenden Modell von Diskurs- bzw. Textkompetenz MeGaDisK mit den drei analytisch zu differenzierenden Kompetenzfacetten: Kontextualisierung, Vertextung und Markierung (Quasthoff 2009; Quasthoff 2011). Diese wurden unter Berücksichtigung der jeweiligen Medialität (schriftlich), Gattung (Argumentieren) sowie der konkreten Schreibaufgabe (Persuasion bzw. Exploration) und Textsorte (Brief bzw. Bericht) operationalisiert bzw. in konkrete Items übersetzt. Im Sinne größtmöglicher Konsistenz der Instrumente wurde bei der Erstellung der Kodier-Materialien für die argumentative explorative Schreibaufgabe Bericht an den Kommissar der Intensivstichprobe (TexQu-Ae) auf theoretisch und empirisch besonders aussagekräftige Items aus den umfassenden Auswertungen der argumentativen persuasiven Schreibaufgabe Brief an Christian in der Ausgangsstichprobe (TexQu-Ap) zurückgegriffen.

\subsubsection{Familiale Interaktionsmuster}

Als familiale Interaktionsmuster wurden die drei Muster berücksichtigt, welche auf Grundlage der qualitativen Analysen mündlicher Eltern-Kind-Interaktionen im Kontext von FUnDuS gesprächsanalytisch rekonstruiert werden konnten (siehe Abschnitt 2.1): Fordern und Unterstützen, Raumlassen und Akzeptieren und Übergehen und Selberlösen.

\footnotetext{
5 Die Auswertungen erfolgten durch geschulte Kodierer auf Basis detaillierter Manuale, in denen jedes Item beschrieben und mit Textbeispielen illustriert ist. Zur Sicherstellung der Reliabilität wurden im Korpus der Ausgangsstichprobe (Brief an Christian) jeweils 10\% eines Jahrgangs doppelt kodiert, im Korpus der Intensivstichprobe alle Texte (Bericht an den Kommissar). Die entsprechenden Indizes fallen für den gesamten Erhebungszeitraum sowohl auf Item- als auch auf Skalenebene durchweg zufriedenstellend aus: Brief an Christian: Kappa $M=0.88, S D=0.17$; ICC $_{3,1} M=0.96, S D=0.06 ;$ ICC $_{3, \mathrm{~K}} M=0.97, S D=0.04$, Bericht an den Kommissar: Kappa $M=0.88$, $S D=0.17 ; \mathrm{ICC}_{3, \mathrm{~K}} M=0.90, S D=0.15$, jeweils gemittelt über drei Erhebungszeitpunkte.
} 
Angesichts der sich empirisch abzeichnenden Stabilität der Muster über die Zeit (Heller \& Krah 2015) und zur besseren Vergleichbarkeit mit den anderen berücksichtigten familialen bzw. individuellen Ressourcen (s. u.) wurden hier ausschließlich die Zuordnungen der Familien bzw. Kinder vom ersten Erhebungszeitraum genutzt.

\subsubsection{Weitere familiale und individuelle Ausgangsbedingungen}

Zur Erfassung weiterer einschlägiger Ausgangsbedingungen wurden der sozioökonomische Status der Familie nach dem International Socio-Economic Index of Occupational Status (ISEI-Index; Ganzeboom, De Graaf \& Treiman 1992) herangezogen sowie Ergebnisse des kognitiven Fähigkeitstests (KFT 4-12 + R; Heller \& Perleth 2000) der Kinder. Beides wurde zu Beginn des Untersuchungszeitraums auf Basis von Fragebogenangaben erfasst.

\subsubsection{Stichprobe}

Diese Datenkonstellation liegt für insgesamt $N=33$ Schülerinnen und Schüler vor, was im Wesentlichen der sog. Intensivstichprobe von FUnDuS entspricht. Diese wurde aus der sog. Ausgangsstichprobe von ca. $N=1500$ Schülerinnen und Schülern über die Kombination von Schülerleistungen (aggregiertes Maß für Argumentationskompetenz $(\mathrm{AK})^{6}$ ) und Angaben zum sozialen Hintergrund der Familie (sozioökonomischer Status (SES)) systematisch gezogen. Der Annahme folgend, dass der SES ein zentraler Prädiktor für Schulleistungen und damit auch für AK ist (Baumert et al. 2006; Conger \& Donnellan 2007; PISA-Konsortium Deutschland 2007), wurden dabei vier mögliche Kombinationen bzw. Gruppen gebildet, die erwartungskonforme (AK und SES hoch bzw. niedrig) und erwartungsdiskrepante (AK hoch und SES niedrig bzw. vice versa) Fälle umfassen. Durch dieses Sampling-Verfahren ist u. a. sichergestellt, dass die Stichprobe trotz der relativ geringen Fallzahl in Hinblick auf die hier berücksichtigten familialen bzw. individuellen Ausgangsbedingungen (SES und kognitive Fähigkeiten) gleichmäßig zusammengesetzt ist.

6 Dieses Maß umfasst sowohl kognitive als auch sprachliche Fähigkeiten: schlussfolgerndes Denken, Wortschatz, Gattungswissen und Indikatoren für schriftliches Argumentieren (Krah et al. 2013; Quasthoff et al. 2012; Quasthoff et al. 2015). 


\subsection{Methodisches Vorgehen}

Die Resultate der linguistischen Textkodierungen sowie der qualitativen Analysen mündlicher familialer Interaktionsmuster wurden zunächst in ein statistisch auswertbares Format überführt ${ }^{7}$ und mit den weiteren familien- bzw. personenbezogenen FUnDuS-Daten der Probanden gematcht.

Zur Beantwortung der formulierten empirischen Forschungsfrage (siehe 3.1) wurde eine Varianzanalyse mit Messwiederholung durchgeführt, in der die Entwicklung der schriftlichen argumentativen Textqualität (abhängige Variablen) für die nach familialen Interaktionsmustern gruppierten Schüler (Faktoren) unter Kontrolle des sozioökonomischen Status' sowie der kognitiven Vorläuferfähigkeiten (Kovariaten) berechnet wurde.

Da dabei jeweils nur Fälle berücksichtigt wurden, bei denen für alle drei Messzeitpunkte Textproduktionsdaten vorlagen, ergeben sich für die Auswertungen der verschiedenen Schreibaufgaben zum Teil unterschiedliche Probandenzahlen (s. u.).

Außerdem dürfen die folgenden Analysen aufgrund der insgesamt geringen Stichproben- bzw. Gruppengröße nur als erste empirische Tendenzen interpretiert werden, welche zukünftig weiterer Absicherung bzw. Überprüfung bedürfen.

\section{Empirische Befunde: Die Entwicklung schriftlicher argumentativer Textproduktion im Verlauf der Sekundarstufe I in Abhängigkeit familialer Interaktionsmuster}

Zunächst sollen nun die Entwicklungsverläufe der einzelnen Gruppen, die jeweils ein Interaktionsmuster darstellen, getrennt für die beiden Aufgabentypen (persuasive und explorative Schreibaufgabe) berichtet werden. Im folgenden Abschnitt werden diese Ergebnisse dann interpretiert und mögliche Erklärungsansätze diskutiert.

7 Für die Auswertung der argumentativen Texte auf Basis der ursprünglich dichotomen Items von TexQu wurde jeweils ein Mittelwert als metrische Variable gebildet, die Interaktionsmuster wurden als kategoriale Variable erfasst. 


\subsection{Bericht der Ergebnisse}

Für die persuasive Schreibaufgabe der Ausgangsstichprobe Brief an Christian $(N=23)$ zeigen sich folgende Befunde (Abb. 3): Kinder aus Familien, welche fordern und unterstützen (FU) bzw. raumlassen und akzeptieren (RA), können das relativ hohe Kompetenzniveau zu Beginn der Erhebung $(M=.35, S D=.08$ bzw. $M=.36, S D=.08)$ im Verlauf der Sekundarstufe I leicht ausbauen $(M=.39$, $S D=.08$ bzw. $M=.39, S D=.11)$. Dabei erfolgt der Kompetenzzuwachs zeitlich versetzt: Während Schülerinnen und Schüler des Musters Fordern und Unterstützen sich von Klasse $6 \mathrm{zu}$ Klasse 7 verbessern, geschieht dies bei den Fällen von Raumlassen und Akzeptieren später, von Klasse $7 \mathrm{zu}$ Klasse 9. Die insgesamt deutlich schwächer ausgeprägte Kompetenz von Kindern, deren Eltern übergehen und selberlösen (ÜSL) $(M=.29, S D=.07)$ nimmt im Verlauf der Sekundarstufe zunächst ab $(M=.23, S D=.10)$, um gegen Ende ungefähr auf bzw. leicht über das Ausgangsniveau anzusteigen $(M=.30, S D=.06)$.

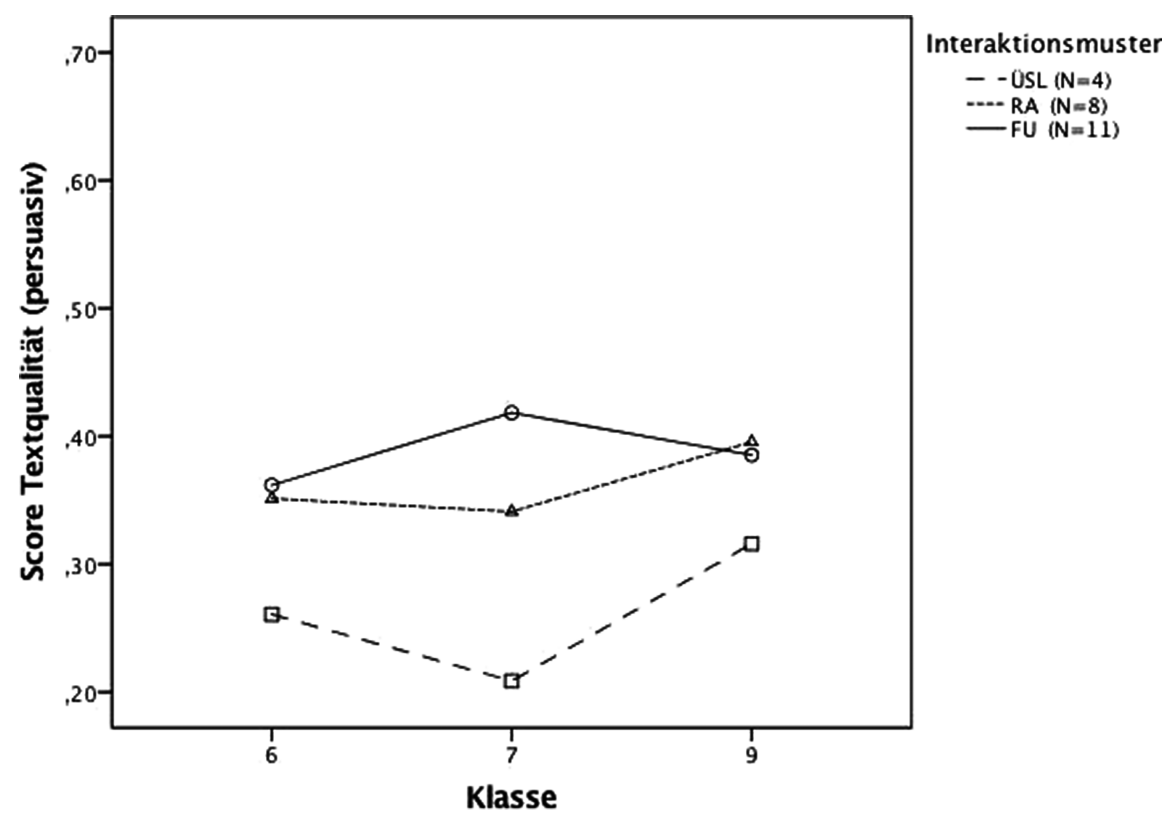

Abb. 3: Entwicklung schriftlicher argumentativer Textproduktion (persuasive Aufgabe) im Verlauf der Sekundarstufe I je nach familialem Interaktionsmuster unter Kontrolle von SES (HISEI $=65.26)$ und kognitiven Vorläuferfähigkeiten (KFT Wortschatztest $=15.65$, logisches Denken $=16.61$ ). 
Es zeigen sich also auch bei Kontrolle einschlägiger Hintergrundvariablen (SES und kognitive Vorläuferfähigkeiten) sehr stabile, nominelle Unterschiede hinsichtlich der Textqualität persuasiver Briefe bzw. ihrer Entwicklung (Kompetenzniveaus bzw. Veränderungen) von Klasse 6 bis Klasse 9, je nachdem, welches Interaktionsmuster in der Familie zu Beginn der Erhebung dominiert. Diese Befunde erweisen sich jedoch weder mit Blick auf die Kompetenzveränderungen der Gruppen über die einzelnen Erhebungszeitpunkte (Haupteffekt Klasse/Alter $F$ $(2,34)=1.008, p=.376$, partielles $\eta 2=.056)$ noch mit Blick auf mögliche differenzielle Entwicklungsverläufe je nach Interaktionsmuster (Interaktionseffekt Klasse/ Alter * Interaktionsmuster $F(4,34)=1.144, p=.352$, partielles $\eta 2=.119)$ als statistisch bedeutsam.

Für die explorative Schreibaufgabe der Intensivstichprobe Bericht an den Kommissar $(N=30)$ zeigen sich folgende Befunde (Abb. 4): Kinder aus Familien, welche fordern und unterstützen (FU), können das bereits hohe Kompetenzniveau in Klasse $6(M=.53, S D=.19)$ im Verlauf der Sekundarstufe I stetig ausbauen und erreichen in Klasse 9 mit Abstand die höchsten Werte $(M=.65, S D=.20)$. Schüle-

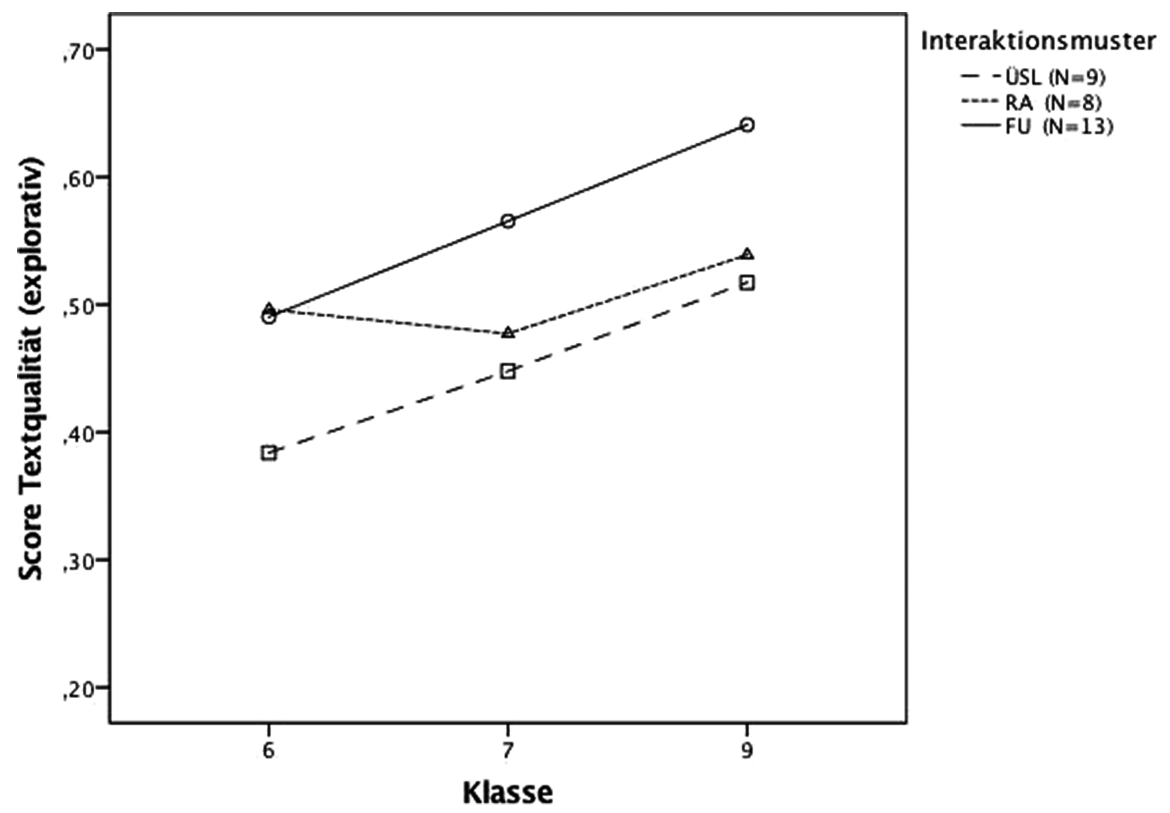

Abb. 4: Entwicklung schriftlicher argumentativer Textproduktion (explorative Aufgabe) im Verlauf der Sekundarstufe I je nach familialem Interaktionsmuster unter Kontrolle von SES $($ HISEI $=57.87)$ und kognitiven Vorläuferfähigkeiten (KFT Wortschatztest $=14.87$, logisches Denken $=15.87$ ). 
rinnen und Schüler, deren Eltern raumlassen und akzeptieren (RA), starten mit ähnlich guten Fähigkeiten $(M=.48, S D=.23)$, zeigen jedoch quasi keine bzw. nur leichte Verbesserungen über den Erhebungszeitraum $(M=.54, S D=.10)$. Anders die Kompetenz von Kindern, deren Eltern übergehen und selberlösen (ÜSL), welche zwar verhältnismäßig schwach ausgeprägt ist $(M=.34, S D=.14)$, jedoch im Verlauf der Sekundarstufe auch kontinuierlich zunimmt $(M=.51, S D=.18)$.

Auch für diese Schreibaufgabe zeigen sich also deutliche Differenzen auf Ebene der Textqualität der verfassten Berichte bzw. ihrer Entwicklung (Kompetenzniveaus bzw. Veränderungen) in Abhängigkeit davon, welches Interaktionsmuster die Eltern zu Beginn der Untersuchung mit ihren Kindern praktizieren und unabhängig vom SES der Familie bzw. den kognitiven Vorläuferfähigkeiten der Schülerinnen und Schüler. Erneut bleibt jedoch auch festzuhalten, dass diese Unterschiede zwar nominell, jedoch nicht statistisch bedeutsam sind (Haupteffekt Klasse/Alter $F(2,48)=0.969, p=.387$, partielles $\eta 2=.039$ und Interaktionseffekt Klasse/Alter * Interaktionsmuster $F(4,48)=0.309, p=.871$, partielles $\eta 2=.025$ ).

\subsection{Interpretation der Ergebnisse}

Betrachtet man die longitudinalen Ergebnisse für beide Schreibaufgaben im Detail, so sind sowohl Ähnlichkeiten als auch Unterschiede zu konstatieren - sowohl mit Blick auf die Kompetenzniveaus der verschiedenen Interaktionsmuster-Gruppen als auch deren Entwicklungsverläufe. Diese werden im Folgenden näher betrachtet sowie mögliche Erklärungsansätze diskutiert.

\subsubsection{Kompetenzniveaus zu Beginn der Erhebung}

Aufgabenübergreifend (Abb. 3 und 4) ,starten“ Kinder aus Familien, welche fordern und unterstützen bzw. raumlassen und akzeptieren, mit einem vergleichbar hohen Kompetenzniveau im schriftlichen Argumentieren. Dieses liegt außerdem deutlich über dem von Schülerinnen und Schülern, deren Eltern in der gemeinsamen Interaktion übergehen und selberlösen.

Dies könnte zum einen auf eine teilweise Assoziation des elterlichen Gesprächsverhaltens mit der jeweiligen Kompetenzausprägung des Kindes deuten. So scheint es zumindest bei argumentativ schwach ausgeprägten Fähigkeiten wahrscheinlich, dass Eltern das Muster Übergehen und Selberlösen praktizieren. Bei Schülerinnen und Schülern mit relativ hoher Argumentationskompetenz scheint es hingegen weniger eindeutig: Hier kommen sowohl das Muster Fordern 
und Unterstützen als auch Raumlassen und Akzeptieren in den Daten vor bzw. in Frage (siehe auch Heller \& Krah 2015).

Zum anderen könnte diese Datenlage auch auf einen kumulativen Effekt der verschiedenen Muster für die kindliche Kompetenzentwicklung hinweisen, der lange vor dem Untersuchungszeitraum des Projekts eingesetzt hat. Gerade angesichts der unterschiedlichen Erwerbssupportivität dieser familialen Interaktionsmuster (Heller \& Krah 2015) wäre es im Sinne einer Henne-Ei-Problematik denkbar, dass zumindest in den eindeutigen bzw. extremen Konstellationen fordernder und unterstützender vs. übergehender und selberlösender Eltern die divergierende Kompetenzausprägung der Kinder zu Beginn der Erhebung bereits Ergebnis langjähriger erwerbsförderlicher vs. -hindernder kommunikativer Erfahrungen bzw. externer Ressourcen (Quasthoff 2011) zu Hause ist.

Derartige Fragen ließen sich nur durch weitere Untersuchungen klären, welche sowohl mögliche Kombinationen kindseitiger Kompetenz und elternseitigen Gesprächsverhaltens als auch deren Ontogenese genau in den Blick nehmen.

\subsubsection{Entwicklungsverläufe der Gruppe Raumlassen und Akzeptieren}

Die Gruppe der Kinder, deren Eltern das Muster Raumlassen und Akzeptieren praktizieren, zeigt über beide argumentative Schreibaufgaben eine sehr ähnliche Entwicklung (Abb. 3 und 4): Während sich die Textqualität von Klasse 6 zu Klasse 7 kaum verändert, steigt sie zu Klasse 9 leicht an, sodass das Niveau am Ende der Erhebung etwas über dem zu Anfang liegt.

Als Erklärungsansatz für diesen Verlauf ist zunächst die - auch im Vergleich zu den anderen Gruppen - hohe Ausgangskompetenz dieser Schülerinnen und Schüler zu berücksichtigen. Möglicherweise befindet diese sich zu Beginn des Untersuchungszeitraums auf einer Art internem Maximalniveau, welches ohne entsprechende familiale kommunikative Unterstützung erst einmal nicht weiter steigerbar ist. Die Tatsache, dass sich die Fähigkeiten nach einer Phase der Stagnation in den folgenden zwei Jahren leicht verbessern, könnte entweder auf die zunehmend stärkere curriculare Berücksichtigung argumentativer Texte gegen Ende der Sekundarstufe $I^{8}$ zurückzuführen sein oder/und darauf, dass die Fami-

8 Vgl. hierzu bspw. die Bildungsstandards für das Fach Deutsch, welche argumentative Tätigkeiten in der Primarstufe ausschließlich im Kompetenzbereich „Sprechen und Zuhören“ verorten (KMK 2005), für den mittleren Schulabschluss jedoch auch im Bereich „Texte schreiben“ (KMK 2004a). 
lie in diesem Alter zunehmend auch von anderen Erwerbsressourcen abgelöst wird, bspw. durch die Interaktion mit Peers (Morek 2014, in diesem Band; Morek \& Quasthoff 2017) oder den eigenen Umgang mit relevanten zielsprachlichen Strukturen (Domenech 2019).

\subsubsection{Entwicklungsverläufe der Gruppen Fordern und Unterstützen und Übergehen und Selberlösen}

In Bezug auf die Entwicklungsverläufe der Interaktionsmuster-Gruppen Fordern und Unterstützen und Übergehen und Selberlösen lassen sich im Vergleich der beiden Schreibaufgaben Unterschiede feststellen: Im Fall des argumentativ-persuasiven Briefs an Christian (Abb. 3) verbessern sich die zu Beginn bereits sehr starken Schülerinnen und Schüler fordernder und unterstützender Eltern von Klasse 6 zu Klasse 7 zunächst, bleiben dann aber bis Klasse 9 mehr oder weniger auf diesem Deckenniveau stehen. Kinder, die zu Hause das Muster Übergehen und Selberlösen erfahren, ,starten' nicht nur mit deutlich schwächeren Fähigkeiten in Klasse 6, sondern verschlechtern sich zu Klasse 7 erst, bevor die Textqualität anschließend bis Klasse 9 relativ steil ansteigt. Ein ganz anderes Bild ergibt sich dagegen bei dem argumentativ-explorativen Bericht an den Kommissar (Abb. 4): Hier verbessern sich beide Gruppen kontinuierlich über den gesamten Erhebungszeitraum quasi parallel auf unterschiedlichen Kompetenzniveaus.

Vor dem Hintergrund obiger Ausführungen (Abschnitt 4.2.2) zum Zusammenspiel von hoher bzw. niedriger Erwerbssupportivität der Muster einerseits sowie der mit steigendem Alter zunehmenden Relevanz außerfamiliärer Erwerbskontexte andererseits lassen sich diese unterschiedlichen Verläufe zusammenfassend als eher erwartungskonform für die persuasive Aufgabe Brief an Christian und als eher erwartungsdiskrepant für die explorative Aufgabe Bericht an den Kommissar zusammenfassen. Hierfür können folgende Erklärungsansätze herangezogen werden:

Zunächst ist zu vermuten, dass das Aufgabenformat der Intensivstichprobe, welches exploratives bzw. abwägendes Argumentieren mit der Textsorte Bericht kombiniert, curricular möglicherweise stärker berücksichtigt wird als der persuasiv angelegte, persönliche Brief der Ausgangsstichprobenerhebung. In diese Richtung weisen bspw. entsprechende Passagen der aktuellen Kernlehrpläne für das Fach Deutsch des Landes Nordrhein-Westfalen: ${ }^{9}$ Über alle Schulformen (Hauptschule,

9 Im Internet einsehbar unter: http://www.schulentwicklung.nrw.de/lehrplaene/lehrplannavi gator-s-i/index.html (12.04.2017). 
Gesamtschule, Realschule, Gymnasium) verändern sich die Kompetenzerwartungen an die argumentative Textproduktion von den Jahrgangsstufen 6 zu 8 und 10 von stärker persönlichen Formen, wie der Textsorte Brief oder/und dem begründenden Darstellen einer bzw. der eigenen Meinung, hin zu stärker sachorientierten, abwägenden Auseinandersetzungen mit verschiedenen Positionen.

Darüber hinaus ist die Schreibaufgabe der Intensivstichprobe im Vergleich insgesamt als profilierter (Bachmann \& Becker-Mrotzek 2010) und für die Schülerinnen und Schüler als motivierender einzuschätzen. So wird bspw. das für den Text notwendige Wissen der zu berücksichtigenden Indizien bzw. Szenarien für den jeweiligen Fall in detaillierter Form bereitgestellt und über die verschiedenen Erhebungszeitpunkte inhaltlich minimal variiert - bei gleichzeitiger Konstanthaltung wesentlicher Punkte der Aufgabe.

Schließlich könnten die festgestellten Unterschiede zwischen den Entwicklungsverläufen der Interaktionsmuster-Gruppen Fordern und Unterstützen und Übergehen und Selberlösen in den beiden Schreibaufgaben auch auf das Setting der Textproduktion selbst zurückzuführen sein. Im Vergleich zu der relativ anonymen Situation der Ausgangserhebung verlangen die Bedingungen der Intensiverhebung von Kindern und Eltern in viel stärkerem Maße, alle verfügbaren Ressourcen einzusetzen. Zudem wussten die Schülerinnen und Schüler ab der zweiten Erhebung, dass sie ihren Text später noch einmal gemeinsam überarbeiten würden, was möglicherweise zu einer von vorneherein höheren Textqualität geführt hat.

In diese Richtung weisen bspw. die folgenden Transkriptausschnitte. So beginnt die Tochter einer Familie des Musters Fordern und Unterstützen in Beispiel 4 Gründe zu generieren, um ihre Version des Tathergangs noch wasserdichter zu machen - ohne dass es dafür durch ihre Mutter eine Veranlassung gegeben hätte:

Bsp. 4 Fall 100 UE 2

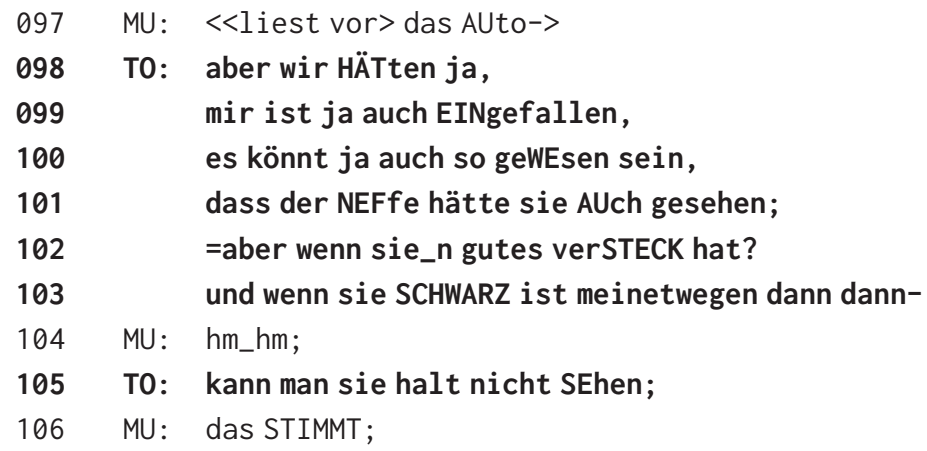


Ebenfalls in einer Dyade der Gruppe Fordern und Unterstützen findet sich der Fall, dass das Kind seinen bereits geschriebenen Text als gute Argumentation gegenüber dem Elternteil verteidigt:

\section{Bsp. 5 Fall 1220 UE 2}

216 MU: und würd die nicht in_n KELler schließen;

217 SO: WEIß man nicht;

218 MU: und WENN sie_s tät,

219 würd sie vielleicht die polizEI nicht anrufen;

220 So: ja das hab ich ja AUCH geschrieben;

Hier statuiert der Sohn durch „weiß man nicht“ (Z. 217), dass die Begründungsgrundlage seiner Mutter nicht stichhaltiger ist als seine eigene, da in beiden Fällen etwas zum schriftlich vorgegebenen Szenario ,hinzugedichtet‘ wurde. Gleichzeitig etabliert er sich damit auch als Wissender über die Textaufgabe, indem er darauf verweist, dass er den Aspekt der Mutter bereits aufgegriffen hat (Z. 220), und stellt seine Version damit als angemessen dar.

Ähnliche Mechanismen finden sich auch in Familien, in denen das Interaktionsmuster Übergehen und Selberlösen Verwendung findet:

\section{Bsp. 6 Fall 1084 UE 2}

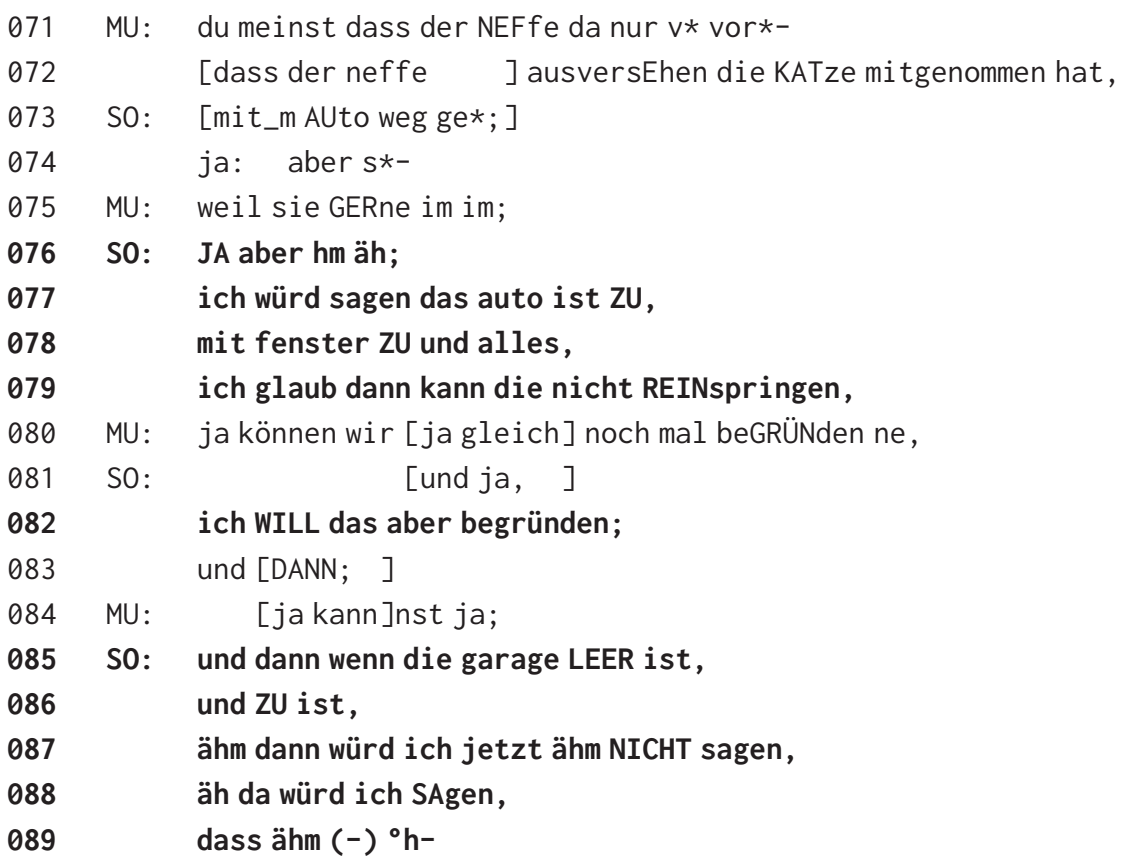


Hier zeigt sich das Engagement des Kindes auf unterschiedliche Art und Weise. So verteidigt der Sohn seinen eigenen Text, indem er bestimmte Mutmaßungen zum Szenario ausspricht, die seine Argumentation stützen: Das Fenster im Auto war zu, die Katze kann nur aus Versehen mitgenommen worden sein (Z. 076-079). Darüber hinaus beharrt er darauf, direkt eine Begründung geben zu dürfen, die seine Argumentation stichfester machen (Z. 082) und führt dies eigeninitiativ sowie in elaborierter Form durch: mit Hilfe einer konsekutiven „wenn dann“-Konstruktion und einer zusätzlichen Begründung (Z. 085-091).

Zusammenfassend spricht also Einiges dafür, dass den Schülerinnen und Schülern im Fall des Berichts an den Kommissar mehr relevantes Wissen für die Textproduktion zur Verfügung stand und sie sich bei dieser (profilierten und auch im Längsschnitt motivierenden) Aufgabe bzw. in diesem (intensiven) Erhebungssetting mehr angestrengt haben. ${ }^{10}$ Dies könnte zu der zunächst erwartungsdiskrepanten, linearen Verbesserung der Textqualität in den Interaktionsmuster-Gruppen Fordern und Unterstützen und Übergehen und Selberlösen über den gesamten Untersuchungszeitraum geführt haben.

\section{Rückblick \& Ausblick}

Ziel dieses Beitrags war es, das Zusammenspiel mündlicher familialer Interaktionsmuster und schriftlicher argumentativer Textproduktion im Verlauf der Sekundarstufe I empirisch zu untersuchen. Vor dem Hintergrund der durchgeführten empirischen Analysen werden im Folgenden zentrale Punkte rückblickend festgehalten sowie offene bzw. sich anschließende Aspekte für zukünftige Arbeiten ausblickend skizziert.

\subsection{Rückblick}

Als Datengrundlage der Untersuchungen diente das reichhaltige längsschnittliche Korpus des Forschungsprojekts FUnDuS, aus dem für $(N=33)$ Schülerinnen

10 Hierfür sprechen auch die insgesamt höheren Werte der Textqualität des Berichts an den Kommissar vs. des Briefs an Christian (siehe Abschnitt 4). 
und Schüler der sogenannten Intensivstichprobe folgende Daten ausgewählt wurden:

- Werte für die Textqualität von zwei unterschiedlichen argumentativen schriftlichen Textproduktionen (Brief an Christian persuasiv und Bericht an den Kommissar explorativ) aus den Klassen 6, 7 und 9, welche mit Hilfe des theoriebasiert entwickelten und empirisch überprüften Verfahrens TexQu (Quasthoff \& Domenech 2016) generiert wurden,

- Informationen über das zu Beginn der Erhebung in der jeweiligen Familie dominierende Muster der Eltern-Kind-Kommunikation (Fordern und Unterstützen, Raumlassen und Akzeptieren oder Übergehen und Selberlösen), welche auf Basis mündlicher, videographierter und transkribierter Interaktionen konversationsanalytisch rekonstruiert wurden,

- standardisierte Angaben über den sozioökonomischen Hintergrund der Familie (ISEI-Index) sowie kognitive Vorläuferfähigkeiten der Kinder (KFT 4-12 + R) zu Beginn der Sekundarstufe I.

Die statistischen Analysen (einfaktorielle Varianzanalyse mit Messwiederholung und Kovariaten) zeigen aufgabenübergreifend - auch bei Kontrolle einschlägiger Hintergrundvariablen (SES und kognitive Vorläuferfähigkeiten) nominelle Unterschiede der Textqualität in Bezug auf die Kompetenzniveaus (Fordern und Unterstützen > Raumlassen und Akzeptieren $>$ Übergehen und Selberlösen) sowie die Entwicklungsverläufe der drei Interaktionsmuster-Gruppen. Diese Befunde erweisen sich jedoch nicht als statistisch bedeutsam - weder mit Blick auf die Kompetenzveränderungen der Gruppen über die drei Erhebungszeitpunkte noch hinsichtlich möglicher differenzieller Entwicklungsverläufe.

Aufgabenvergleichend ergibt sich angesichts der unterschiedlichen Erwerbssupportivität der Muster (Heller \& Krah 2015) ein tendenziell erwartungskonformes Bild für den persuasiven Brief an Christian und ein tendenziell erwartungsdiskrepantes für den explorativ angelegten Bericht an den Kommissar. Hier kann die überraschend kontinuierliche bzw. parallele Verbesserung der Textqualität der ,Extremgruppen' Fordern und Unterstützen und Übergehen und Selberlösen jedoch unter Berücksichtigung curricularer und aufgabenbezogener Aspekte sowie der unterschiedlichen Erhebungssettings in der Ausgangs- vs. der Intensivstichprobe teilweise erklärt werden.

Zusammenfassend spricht dies ontogenetisch für die Relevanz häuslicher kommunikativer Erfahrungen als Erwerbsressource für zentrale schulsprachliche Fähigkeiten in der Sekundarstufe I. Didaktisch-methodisch sind die Wichtigkeit einer profilierten und motivierenden Aufgabenkonstruktion sowie die Gestaltung des jeweiligen Textproduktions- bzw. Erhebungssettings festzuhalten. 


\subsection{Ausblick}

Vor dem Hintergrund der Ergebnisse dieses Beitrags mit einer für quantitative Berechnungen kleinen Stichprobe wäre zukünftig zu überprüfen, ob die nominellen Unterschiede der Textqualität bzw. ihrer Entwicklung in Abhängigkeit familialer Interaktionsmuster sich bei einer größeren Stichprobe als statistisch bedeutsam erweisen.

Aus ontogenetischer Perspektive wäre außerdem eine genauere Erforschung der Wechselbeziehung(en) von den verschiedenen häuslichen Interaktionsmustern und kindseitigen Kompetenzentwicklungen anzustreben. So könnten bspw. die derzeit noch offenen Fragen geklärt bzw. ansatzweise beantwortet werden, ob und welche typischen Muster-Kompetenz-Kombinationen es gibt bzw. wie und wann sich diese herausbilden.

Hierbei wäre auch zu berücksichtigen, inwiefern die Ausprägungen mündlicher und schriftlicher argumentativer Fähigkeiten miteinander einhergehen oder nicht. Vorliegende Ergebnisse der Analysen derartiger Zusammenhänge im Bereich argumentativer (Domenech \& Krah 2014), narrativer (Quasthoff, Ohlhus \& Stude 2009) und allgemeinsprachlicher Kompetenzen (Bremerich-Vos, Böhme \& Robitsch 2008; Jude 2008 ${ }^{11}$ ) verweisen zwar zum einen auf ein gewisses Maß an Konvergenz, zum anderen aber auch auf Divergenz bzw. Varianz, bspw. in Abhängigkeit der ,Schriftnähe“ der jeweiligen Gattung im Mündlichen oder individueller Vorerfahrungen (Quasthoff, Ohlhus \& Stude 2009).

Darüber hinaus scheint es vielversprechend, neben dem ,globalen“ Effekt der Interaktionsmuster auf die Textproduktion genauer zu untersuchen, welches Muster sich auf welche der oben (Abschnitt 3.1.1) genannten MeGaDisKFacetten von Diskurs- bzw. Textkompetenz (Quasthoff 2009; Quasthoff 2011) wie auswirkt bzw. die von Heller \& Krah (2015) auf Basis mündlicher Daten formulierten Hypothesen auf das Schriftliche zu übertragen. Demnach sollten sich fordernde und unterstützende Praktiken in der Familie positiv auf alle Kompetenzfacetten auswirken, raumlassende und akzeptierende Erfahrungen insb. auf die Fähigkeit zur Vertextung, während das Verhalten Übergehen und Selberlösen allenfalls Verbesserungen im Bereich der Kontextualisierung erwarten ließe.

Aus Sicht der Schreibforschung wäre es überdies von Interesse, den Prozess der Textüberarbeitung näher in den Blick zu nehmen. Je nach Datenkonstellation könnten hier entweder Zusammenhänge zwischen der mündlichen Aushandlung und der schriftlichen Ausgestaltung fokussiert oder aber auch implizite

11 Wobei mündliche Fähigkeiten hier nur rezeptiv einbezogen wurden. 
Vorstellungen guter Textqualität bzw. -arbeit bei Eltern oder/und Kindern rekonstruiert werden.

Letztlich unterstreichen die Ergebnisse dieses Beitrags andere Befunde der FUnDuS-Studie, welche wiederholt die Relevanz häuslicher Kommunikation für verschiedene Aspekte argumentativer Kompetenzentwicklung im Verlauf der Sekundarstufe I herausgestellt haben - und zwar unabhängig von anderen prominenten Prädiktoren wie der sozialen Herkunft oder individueller Vorläuferfähigkeiten (Quasthoff et al. 2016). An dem förderlichen Potenzial eben dieser Ressourcen ansetzend wurde in Folge-Projekten bereits ein Elterntraining zur Veränderung familialer Interaktionsmuster (Eltern fördern durch Argumentieren, EfA) erfolgreich entwickelt, durchgeführt und empirisch evaluiert (Quasthoff et al. 2015; Quasthoff et al. 2016; Kluger in diesem Band) und für die Fortbildung mit Fachkräften im Sinne eines Train-the-Trainer-Konzepts adaptiert (EfA-TT). Perspektivisch scheint in diesem Zusammenhang u. a. auch untersuchenswert, inwieweit sich neben den bereits festgestellten Änderungen von Einstellungen bzw. kommunikativen Praktiken auf Elternseite (Quasthoff et al. 2015; Quasthoff et al. 2016) auch Auswirkungen auf (z. B. kognitive, kommunikative, mündliche, schriftliche) Dimensionen kindseitiger Kompetenzentwicklung feststellen lassen.

\section{Literatur}

Augst, Gerhard, Katrin Disselhoff, Alexandra Henrich, Thorsten Pohl, \& Paul-Ludwig Völzing (2007): Text - Sorten - Kompetenz: Eine echte Longitudinalstudie zur Entwicklung der Textkompetenz im Grundschulalter. Frankfurt am Main: Lang.

Bachmann, Thomas \& Michael Becker-Mrotzek (2010): Schreibaufgaben situieren und profilieren. In Thorsten Pohl \& Torsten Steinhoff (Hrsg.), Textformen als Lernformen, 191-209. Duisburg: Gilles \& Francke.

Baumert, Jürgen, Petra Stanat \& Rainer Watermann (2006): Herkunftsbedingte Disparitäten im Bildungswesen: Differenzielle Bildungsprozesse und Probleme der Verteilungsgerechtigkeit. Vertiefende Analysen im Rahmen von PISA 2000. Wiesbaden: VS Verlag für Sozialwissenschaften.

Becker-Mrotzek, Michael \& Ingrid Böttcher (2012): Schreibkompetenz entwickeln und beurteilen. Praxishandbuch für die Sekundarstufe I und II. 4. Auflage. Berlin: Cornelsen-Scriptor.

Bremerich-Vos, Albert, Katrin Böhme \& Alexander Robitzsch (2008): Sprachliche Kompetenzen im Fach Deutsch - Strukturanalysen und Validierungsbefunde. In Dietlinde Granzer, Olaf Köller \& Albert Bremerich-Vos (Hrsg.), Bildungsstandards Deutsch und Mathematik: Leistungsmessung in der Grundschule, 198-218. Weinheim: Beltz.

Conger, Rand \& Brent Donnellan (2007): An interactionist perspective on the socioeconomic context of human development. Annual Review of Psychology 58, 175-199. 
Domenech, Madeleine (2019): Schriftsprachliche Profile von Fünftklässlern: Argumentative Briefe im Zusammenspiel unterschiedlicher textueller, familiärer und individueller Ressourcen. Berlin: De Gruyter.

Domenech, Madeleine, Vivien Heller \& Inger Petersen (2018): Argumentieren mündlich, schriftlich, zweitsprachlich: Verfahren und Anforderungen. Beiträge zur Fremdsprachenvermittlung 26, 15-35.

Domenech, Madeleine \& Antje Krah (2014): What familial aspects matter? Investigating argumentative competences of learners at the beginning of secondary schooling in the light of family-based resources. Learning, Culture and Social Interaction 3(2), 77-87.

Domenech, Madeleine, Antje Krah \& Jelena Hollmann (2017): Entwicklung und Förderung argumentativer Fähigkeiten in der Sekundarstufe I: Die Relevanz familiärer Ressourcen. Bildung und Erziehung, 70(1), 91-107.

Ehlich, Konrad, Renate Valtin \& Beate Lütke (2012): Expertise Erfolgreiche Sprachförderung unter Berücksichtigung der besonderen Situation Berlins. Berlin: Senatsverwaltung für Bildung, Jugend und Wissenschaft.

Feilke, Helmuth (2008): Schriftlich argumentieren - Kompetenzen und Entwicklungsbedingungen. In Eva Burwitz-Melzer, Wolfgang Hallet, Michael Legutke, Franz-Joseph Meißner \& Joybrato Mukherjee (Hrsg.), Sprachen lernen - Menschen bilden. Dokumentation zum 22.Kongress für Fremdsprachendidaktik der Deutschen Gesellschaft für Fremdsprachenforschung (DGFF), 153-164. Baltmannsweiler: Schneider-Verlag Hohengehren.

Feilke, Helmuth (2013): Bildungssprache und Schulsprache am Beispiel literal-argumentativer Kompetenzen. In Michael Becker-Mrotzek, Karen Schramm, Eike Thürmann, \& Helmut J. Vollmer (Hrsg.), Sprache im Fach: Sprachlichkeit und fachliches Lernen, 113-130. Münster: Waxmann.

Ganzeboom, Hary B. G., Paul M. De Graaf \& Donald J. Treiman (1992): A Standard International Socio-Economic Index of Occupational Status. Social science research 21(1), 1-56.

Hausendorf, Heiko \& Uta Quasthoff (1996): Sprachentwicklung und Interaktion: Eine linguistische Studie zum Erwerb von Diskursfähigkeiten bei Kindern. Wiesbaden: Westdeutscher Verlag/Radolfzell: Verlag für Gesprächsforschung.

Hausendorf, Heiko \& Uta M. Quasthoff (2005): Konversations-/Diskursanalyse: (Sprach-)Entwicklung durch Interaktion. In Günter Mey (Hrsg.), Qualitative Forschung in der Entwicklungspsychologie, 585-618. Köln: Kölner Studien Verlag.

Heller, Kurt A. \& Christoph Perleth (2000): Kognitiver Fähigkeitstest für 4. bis 12. Klassen, Revision: KFT 4-12+R. Göttingen: Beltz Test.

Heller, Vivien (2012): Kommunikative Erfahrungen von Kindern in Familie und Unterricht. Passungen und Divergenzen. Tübingen: Stauffenburg.

Heller, Vivien \& Antje Krah (2015): Wie Eltern und Kinder argumentieren. Interaktionsmuster und ihr erwerbssupportives Potenzial im längsschnittlichen Vergleich. Mitteilungen des Deutschen Germanistenverbandes 62 (1), 5-20.

Hollmann, Jelena, Elke Wild, Uta Quasthoff, Antje Krah \& Nantje Otterpohl (2012): Der Erwerb sprachlicher Kompetenzen im Elternhaus: Erste Befunde zur Güte eines Fragebogens zur Erfassung des Anregungsgehalts von Familienkonversationen. Unterrichtswissenschaft 40(1), 47-63.

Jude, Nina (2008): Zur Struktur von Sprachkompetenz. Frankfurt am Main: www.dissonline.de. Im Internet abrufbar unter: http://publikationen.ub.uni-frankfurt.de/volltexte/2009/ 6794/pdf/ Jude_Zur_Struktur_von_Sprachkompetenz.pdf (28.04.2017). 
KMK (2004a): Bildungsstandards im Fach Deutsch für den Mittleren Schulabschluss: Beschluss vom 4. 12.2003.München: Wolters Kluwer. http://www.kmk.org/fileadmin/ veroeffentlichungen_beschluesse/2003/2003_12_04-BS-Deutsch-MS.pdf (28.04.2017). KMK (2004b) Bildungsstandards im Fach Mathematik für den Mittleren Schulabschluss: Beschluss vom 4. 12.2003.München: Wolters Kluwer. http://www.kmk.org/fileadmin/ veroeffentlichungen_beschluesse/2003/2003_12_04-Bildungsstandards-Mathe-Mittle ren-SA.pdf (28.04.2017).

KMK (2005): Bildungsstandards im Fach Deutsch für den Primarbereich: Beschluss vom 15.10.2004. München: Wolters Kluwer.

Krah, Antje, Uta Quasthoff, Vivien Heller, Elke Wild, Jelena Hollmann \& Nantje Otterpohl (2013): Die Rolle der Familie beim Erwerb komplexer sprachlicher Fähigkeiten in der Sekundarstufe I. In Angelika Redder \& Sabine Weinert (Hrsg.), Sprachförderung und Sprachdiagnostik. Interdisziplinäre Perspektiven, 68-88. Münster: Waxmann.

Krelle, Micheal \& Heiner Willenberg (2008): Argumentation Deutsch. In DESI-Konsortium (Hrsg.), Unterricht und Kompetenzerwerb in Deutsch und Englisch. Ergebnisse der DESI- Studie, 81-88. Weinheim: Beltz.

Morek, Miriam (2012): Kinder erklären. Interaktionen in Familie und Unterricht im Vergleich. Tübingen: Stauffenburg.

Morek, Miriam (2014): Constructing social and communicative worlds - The role of peerinteractions in preadolescents' discursive development. Learning, Culture and Social Interaction 2(3), 121-133.

Morek, Miriam \& Vivien Heller (2012): Bildungssprache - kommunikative, epistemische, soziale und interaktive Aspekte ihres Gebrauchs. Zeitschrift für angewandte Linguistik 57, 67-101.

Morek, Miriam \& Uta Quasthoff (2017): Sprachliche und diskursive Praktiken unter Kindern. In Eva Neuland \& Peter Schlobinski (Hrsg.), Handbuch Sprache in sozialen Gruppen. Handbücher Sprachwissen, 255-275. Berlin: De Gruyter.

Neumann, Astrid \& Rainer Lehmann (2008): Schreiben Deutsch. In DESI-Konsortium (Hrsg.), Unterricht und Kompetenzerwerb in Deutsch und Englisch, 89-103. Weinheim: Beltz.

PISA-Konsortium Deutschland (Hrsg.) (2007): PISA 2006. Die Ergebnisse der dritten internationalen Vergleichsstudie. Münster: Waxmann.

Pohl, Thorsten (2014): Schriftliches Argumentieren. In Helmut Feilke \& Thorsten Pohl (Hrsg.), Schriftlicher Sprachgebrauch. Texte verfassen. Deutschunterricht in Theorie und Praxis. 287-315. Baltmannsweiler: Schneider Verlag Hohengehren.

Quasthoff, Uta (2009): Entwicklung der mündlichen Kommunikationskompetenz. In Michael Becker-Mrotzek (Hrsg.), Unterrichtskommunikation und Gesprächsdidaktik. Deutschunterricht in Theorie und Praxis, 84-100. Baltmannsweiler: Schneider Verlag Hohengehren.

Quasthoff, Uta (2011): Diskurs- und Textfähigkeiten. Kulturelle Ressourcen ihres Erwerbs. In Ludger Hoffmann, Kerstin Leimbrink \& Uta Quasthoff (Hrsg.), Die Matrix der menschlichen Entwicklung, 210-251. Berlin, Boston: De Gruyter.

Quasthoff, Uta \& Madeleine Domenech (2016): Theoriegeleitete Entwicklung und Überprüfung eines Verfahrens zur Erfassung von Textqualität (TexQu) am Beispiel argumentativer Briefe in der Sekundarstufe I. Didaktik Deutsch 41, 21-43.

Quasthoff, Uta \& Friederike Kern (2007): Familiale Interaktionsmuster und kindliche Diskursfähigkeit: Mögliche Auswirkungen interaktiver Stile auf diskursive Praktiken und Kompetenzen bei Schulkindern. In Hausendorf, Heiko (Hrsg.), Gespräch als 
Prozess. Linguistische Aspekte der Zeitlichkeit verbaler Interaktion, 277-306. Tübingen: Gunter Narr.

Quasthoff, Uta \& Antje Krah (2015): Familiale Kommunikation als Spracherwerbsressource: Das Beispiel argumentativer Kompetenzen. In Eva Neuland (Hrsg.), Sprache der Generationen, 127-144. Frankfurt am Main u. a.: Peter Lang.

Quasthoff, Uta, Sören Ohlhus \& Juliane Stude (2009): Der Erwerb von Textproduktionskompetenz im Grundschulalter: Ressourcen aus der Mündlichkeit und ihre unterschiedliche Nutzung. Zeitschrift für Grundschulforschung 2(2), 56-68.

Quasthoff, Uta, Elke Wild, Vivien Heller, Jelena Hollmann, Antje Krah, Sören Ohlhus, Nantje Otterpohl \& Petra Strähle (2012): FUnDuS I. Unveröffentlichter inhaltlicher Abschlussbericht. https://www.tib.eu/de/suchen/id/TIBKonversationsanalyseT\% 3A782845975/Abschlussbericht-FUnDuS-Projekt-Forschungsprojekt/?tx_tibsearch_ search $\% 5$ Bsearchspace $\% 5 D=$ tn

Quasthoff, Uta, Elke Wild, Madeleine Domenech, Jelena Hollmann, Christian Kluger, Antje Krah, Nantje Otterpohl \& Johannes Wibbing (2015): Mündliches und schriftliches Argumentieren in der Sekundarstufe I. Können Eltern ihre Kinder beim Erwerb dieser Schlüsselkompetenz unterstützen? In Angelika Redder, Johannes Naumann \& Rosemarie Tracy (Hrsg.), Forschungsinitiative Sprachdiagnostik und Sprachförderung - Ergebnisse, 207-233. Münster: Waxmann.

Quasthoff, Uta, Elke Wild, Madeleine Domenech, Jelena Hollmann, Christian Kluger, Antje Krah \& Nantje Otterpohl (2016): Abschlussbericht des BMBF-geförderten Projekts „Die Rolle familialer Unterstützung beim Erwerb von Diskurs- und Schreibfähigkeiten in der Sekundarstufe I“ (FUnDuS, 01 GJ 1207A/B) der Universität Bielefeld und der Technischen Universität Dortmund. https://www.tib.eu/de/suchen/id/TIBKonversationsanalyseT\% 3A782845975/Abschlussbericht-FUnDuS-Projekt-Forschungsprojekt/?tx_tibsearch_search $\% 5$ Bsearchspace $\% 5 \mathrm{D}=$ tn

Rezat, Sara (2011): Schriftliches Argumentieren. Zur Ontogenese konzessiver Argumentationskompetenz. Didaktik Deutsch 31, 50-67.

Vollmer, Helmut Johannes (2011): Schulsprachliche Kompetenzen. Zentrale Diskursfunktionen. Osnabrück: Universität Osnabrück. http://www.home.uni-osnabrueck.de/hvollmer/ VollmerDF-Kurzdefinitionen.pdf (18.04.2016).

Wild, Elke, Uta Quasthoff, Jelena Hollmann, Nantje Otterpohl, Antje Krah \& Sören Ohlhus (2012): Die Rolle familialer Unterstützung beim Erwerb von Argumentationskompetenz in der Sekundarstufe I. Diskurs Kindheits- und Jugendforschung 7(1), 101-112.

Winkler, Iris (2003): Argumentierendes Schreiben im Deutschunterricht. Theorie und Praxis. Frankfurt am Main u. a.: Peter Lang. 